\title{
Espondilodiscite causada por Candida parapsilosis: relato de caso e revisão da literatura
}

\section{Spondilodiscit caused by Candida parapsilosis: case report and literature review}

\author{
Leandro da Costa Lane Valiengo', Carlo Emanuel Petitto', Julio \\ Reno Sawada ${ }^{1}$, Ricardo B. V. Fontes ${ }^{2}$, Fernando C. G. Pinto ${ }^{3}$
}

\begin{abstract}
Valiengo LCL, Petitto CE, Sawada JR, Fontes RBV, Pinto FCG. Espondilodiscite causada por Candida parapsilosis: relato de caso e revisão da literatura. Rev Med (São Paulo). 2007 abr.jun.;86(2):112-6.
\end{abstract}

RESUMO: INTRODUÇÃO: São raras as espondilodiscites fúngicas decorrentes de espécies do gênero Candida, sendo que até 2004 foram descritos menos de 75 casos. RELATO DE CASO: Homem, 42 anos, com história de etilismo por 35 anos, foi admitido com quadro de dor em abdome inferior com irradiação para membros inferiores e fraqueza dos mesmos nos últimos 3 meses. Os achados do exame físico foram: atrofia muscular em região glútea, dor à mobilização da articulação coxo-femoral, lombalgia em níveis L4 e L5 e força muscular grau II de parte distal de membros inferiores. O paciente foi internado e introduziu-se empiracamente clindamicina e ciprofloxacina por suspeita de espondilodiscite, confirmada por meio do exame de Ressonância Magnética (RM). Após duas semanas o paciente começou a apresentar quadro de dor à inspiração. A radiografia e a tomografia de tórax mostraram quadro difuso multinodular em pulmão sugestivo de infecção fúngica ou tuberculose de apresentação atípica. A biópsia demonstrou granuloma com necrose central e a cultura Candida albicans. Após 1 mês de antibioticoterapia houve melhora da dor em abdome inferior e membros inferiores porém sem melhora radiológica. Foi então realizada punção aspirativa da massa lombar com cultura positiva para Candida parapsilosis, introduzindo-se Anfoterecina B. Cinco meses após uso contínuo desta droga houve melhora radiológica pulmonar e da coluna lombar, com remissão dos sintomas. DISCUSSÃO: O uso abusivo de álcool constitui um fator de risco para o desenvolvimento de espondilodiscite por espécies do gênero Candida, visto que teoricamente predispõe a ocorrência de infecções sistêmicas por organismos normalmente de baixa virulência. CONCLUSÃO: A espondilodiscite causada por Candida é um evento raro, mas deve ser suspeitada em pacientes com algum tipo de imunosupressão, como o etilismo grave.

DESCRITORES: Discite. Imunossupressão. Alcoolismo. Estudo de caso. Literatura de revisão. Candida/classificação. Candida/patogenicidade.

\footnotetext{
${ }^{1}$ Acadêmicos de Medicina da FMUSP.

${ }^{2}$ Coordenador, Residente Neurocirurgia HCFMUSP.

${ }^{3}$ Coordenador, Assistente Neurocirurgia HCFMUSP.

Endereço para correspondência: Carlo E. Petitto. R. Teodoro Sampaio, 487. Ap. 51, Pinheiros - São Paulo . CEP 05405000.e- mail: carlopetitto@gmail.com
} 


\section{INTRODUÇÃO}

A tualmente, a quarta causa de organismos oriundos de infecção nosocomial isolados em cultura sanguínea nos Estados Unidos é devido a espécies de Candida, perdendo apenas para Staphylococcus aureus, estafilococos coagulase negativos e enterococos, e pode causar doença invasiva em indivíduos com o sistema imunológico reduzido ${ }^{1}$. A freqüência de candidemia nosocomial triplicou entre 1978 e 1984 e dobrou entre 1980 e 1990². Isso pode ser explicado pelo grande aumento do uso cateteres centrais, uso de antibióticos de amplo espectro, uso de medicações imunossupressoras, por doenças neoplásicas e abuso de substâncias endovenosas ${ }^{3,4}$. Infelizmente, agentes antifúngicos eficientes são muito limitados e associados com alguns efeitos adversos graves ${ }^{5}$.

Candida albicans é ainda o organismo mais freqüente das candidemias, entretanto outras espécies estão aumentando de freqüência ${ }^{6-9}$, sendo relatado aqui um caso de Candida parapsilosis.

\section{MÉTODOS}

Foram feitas diversas pesquisas no MEDLINE, na literatura de língua inglesa entre 1966 e agosto de 2005, e uma procura bibliográfica de artigos para identificar casos claramente documentados de espondilodiscites causados pelas espécies de Candida. Foram revisados dados como: quadro clínico, topografia da lesão, resultados de laboratório e terapêutica utilizada. Só foi considerado sendo um caso de espondilodiscite quando havia registro laboratorial, de imagem e quadro clínico compatível com espondilodiscite junto a um diagnóstico de candida em cultura e/ou histologia de biópsia.

\section{Relato de caso}

Homem, 42 anos, com história de etilismo por 35 anos, foi admitido com quadro de dor em quadril com irradiação para membros inferiores e fraqueza dos mesmos nos últimos 3 meses, evoluindo com incapacidade de deambulação. Além disso, referia uma tosse seca diária que havia surgido alguns meses antes. De antecedentes pessoais apresentava hipertensão arterial e pancreatite crônica há 5 anos. Os achados do exame físico foram: atrofia muscular em região glútea, dor à mobilização da articulação coxo-femoral, lombalgia em níveis L4 e L5 e força muscular grau Il de parte distal de membros inferiores. $O$ paciente foi internado e introduziu-se empirlcamente clindamicina e ciprofloxacina por suspeita de espondilodiscite, confirmada por meio do exame de Ressonância Magnética (RM) que demonstrou acometimento dos níveis L4 e L5. O VHS era de $61 \mathrm{~mm} / \mathrm{h}$ e os leucócitos de 14420 células.

Após duas semanas o paciente começou a apresentar quadro de dor à inspiração. A radiografia e a tomografia de tórax mostraram quadro difuso multinodular em pulmão sugestivo de infecção fúngica ou tuberculose de apresentação atípica. $O$ exame citológico do material coletado através de broncoscopia demonstrou, a presença de pseudohifas e esporos fúngicos e sem células neoplásicas. A biópsia demonstrou granuloma com necrose central e a cultura Candida albicans, sendo esperado mais investigação para orientar tratamento. Após um mês de antibioticoterapia houve melhora da dor em quadril e membros inferiores porém sem melhora radiológica. Foi então realizada punção aspirativa da massa lombar com cultura positiva para Candida parapsilosis introduzindo-se Anfoterecina B $50 \mathrm{mg} / \mathrm{d}$, chegandose até a dose de $335 \mathrm{mg} / \mathrm{d}$. Cinco meses após uso contínuo desta droga houve melhora radiológica pulmonar e da coluna lombar, com remissão dos sintomas. Dois anos após o paciente se encontra sem sintomas e em bom estado geral.

\section{RESULTADOS}

As principais características dos 82 pacientes revisados da literatura, junto com o nosso, estão expostos na tabela. A idade média desses pacientes foi 52 anos (indo de meses até 88 anos). A maior parte dos pacientes tinha idade entre 40 e 80 anos (68,6\% dos pacientes). Houve uma proporção maior de homens que mulheres de 1,9:1. O nível mais acometido foi L1-2 (21\% dos casos), seguido de L45 (17,3\%), L3-4 (7,4\%), T9-10 (7,4\%), T12-L1 (6\%), T10-11 (5\%) e T7-8 (3,7\%). A coluna lombar foi a mais comprometida ( $65,4 \%$ dos casos) junto com a torácica $(39,5 \%)$. A sacral foi acometida em apenas $6 \%$ e a cervical em $5 \%$ do total de pacientes. Isso totaliza um acometimento de coluna torácia inferior e lombosacral de $96,4 \%$.

Entre os pacientes com quadro clínico descrito todos apresentavam lombalgia ou dorsalgia, 30\% apresentavam febre, $20 \%$ relatavam perda de peso e $19,6 \%$ tinham déficits neurológicos. Outros sintomas menos comuns foram inespecíficos como anorexia e fraqueza. As principais alterações laboratoriais relatadas são o aumento do VHS que ocorreu em $86,3 \%$ e o aumento da contagem de leucócitos (WBC) que ocorreu em $18,4 \%$, sendo que 
outros exames não tiveram muita importância. A hemocultura veio positiva para candidiase em $50,8 \%$ dos 60 casos relatados desse exame. O diagnóstico foi confirmado por cultura da biópsia (ou autópsia) em todos os casos, exceto 3 pacientes que evidência histológica do acometimento vertebral apenas. Foram relatadas 6 espécies diferentes de Candida, sendo $59,4 \%$ C albicans, $17,7 \%$ de $C$. tropicalis, $13,9 \%$ de C.glabrata, 6,3\% de C.parapsilosis e apenas um paciente com $C$ kefyr e um com G.guiliermondii.

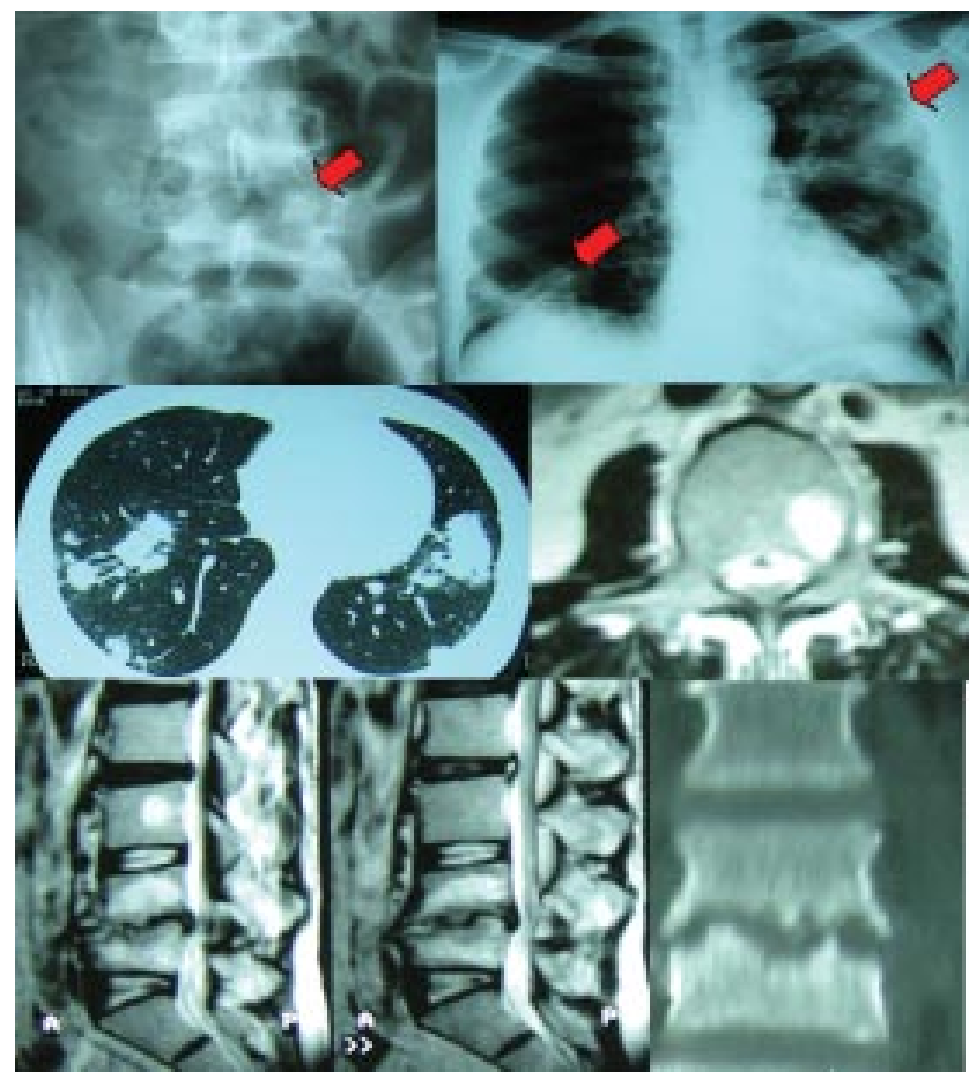

Figura 1. Espondilodiscite e acometimento pulmonar causado por Candida em paciente etilista crônico. Setas vermelhas indicando as lesões em radiografia simples.

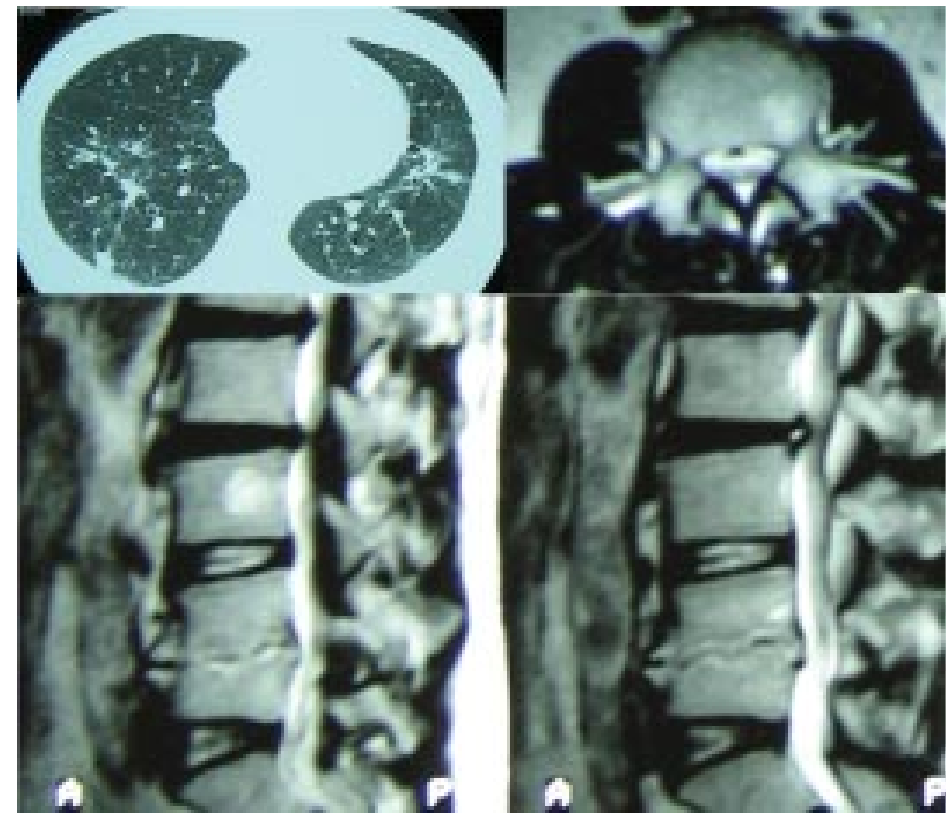

Figura 2. Melhoria dos padrões radiológicos associado a remissão dos sintomas. 
Os principais fatores de risco encontrados para espondilodiscite por Candida foram: uso de cateteres centrais $(46,9 \%)$, uso de antibiótico (42\%) e imunosuprimidos, como HIV, quimioterapia, uso de esteróides, transplante de medula, $(38,2 \%)$ e uso de drogas injetáveis (16,9\%). Outros fatores envolvidos também em infecções de baixa virulência foram cirurgia prévia (21\%), DM (8,6\%), neoplasia (18,5\%) e etilismo $(9,8 \%)$. Além disso, traumas e cirurgias locais foram relatados em $11 \%$ dos casos. Há de se relatar que no grupo entre 19 e 25 anos o principal fator de risco foi o uso de drogas injetáveis presente em $75 \%$ dos casos.

O tratamento consistiu de tratamento medicamentoso e cirúrgico combinado em $38,2 \%$ e apenas medicamentoso em $55,3 \%$ dos casos. O tratamento cirúrgico consistia basicamente de debridamento e fusão vertebral. O medicamento mais utilizado foi anfoterecina B $(79,2 \%)$ e apenas 14 pacientes não a usaram. A dose total foi de 1 a $2 \mathrm{~g} \mathrm{e}$ a duração foi entre 1 a 16 semanas. Sete pacientes usaram a anfoterecina B lipídica. As outras medicações mais usadas foram 5 -flucitosina (28\%) e fluconazol (29,2\%). A terapia com azóis teve uma duração um pouco maior que com anfoterecina $B$, durando por volta de 10 semanas.

O prognóstico da maioria dos pacientes com espondilodiscite por Candida é favorável. Como é mostrado, muitos curam sendo definido isso através de melhora de quadro clínico, radiológico e laboratorial. Entre os casos relatados (apenas 5 não foram) e que teve tempo de ter seguimento (5 não tiveram), $83 \%$ teve cura, 2 casos ficaram com déficits neurológicos e 2 morreram por candidiase invasiva (um com C. tropicalis e outro uma espécie não descrita de Candida). Houve 8 mortes não relacionadas ao quadro de candidíase.

$\mathrm{Em}$ relação aos pacientes apenas com $C$. parapsilosis não há muita mudança em relação às características. Todos os pacientes eram homens, tinham média de idade de 54 anos e houve acometimento lombar e de L4 em todos os 5 casos. Apenas um paciente apresentou hemocultura positiva para espécies de Candida. Além disso, todos receberam fluconazol como tratamento, e dois foram também submetidos a cirurgia e a cura ocorreu em todos os casos. Os fatores de risco não apresentam /diferenças em relação às outras espécies.

\section{DISCUSSÃO}

É muito raro ocorrer infecção fúngica na coluna vertebral. Espécies de Candida geralmente causam infecções benignas e fazem parte da flora normal do organismo. Entretanto, quando há algum grau de disfunção da imunidade do hospedeiro, pode haver invasão patogênica por esse organismo. Nossa revisão mostrou apenas 82 casos de osteomielite causada por Candida sp.

A osteomielite vertebral pode ocorrer por via hematogênica de um foco distante ou por extensão direta de um foco próximo ${ }^{10}$. O elevado suprimento vascular das vértebras e de seus discos explica porque a via hematogênica é a mais comum de disseminação em adultos ${ }^{11}$. Mesmo que apenas $50,8 \%$ dos pacientes tenham hemocultura positiva para Candida sp, é bem provável que a maior parte dos casos tenha ocorrido por disseminação hematogênica. O nosso caso relatado é um exemplo disso, já que a hemocultura veio sem crescimento de organismos e havia 2 focos diferentes de Candida $s p$. A disseminação hematogênica foi demonstrada após ser mostrado haver a mesma genotipagem molecular entre $C$. tropicalis de organismos encontrados no osso e no sangue ${ }^{9}$.

O quadro clínico consiste em lombalgia ou dorsalgia, um terço apresenta febre, 20\% relatam perda de peso assim como déficits neurológicos. Não há nenhum exame laboratorial específico identificado.

O diagnóstico de espondilodiscite por Candida é feito pelo quadro clínico suspeito, mostrando a lesão por um exame de imagem e confirmado através de alguma prova microbiológica. A CT e a radiografia simples podem ser úteis, mas o exame de escolha para espondilodiscite é a MRI ${ }^{12}$. Alguns autores dizem que a ausência de abcesso paravertebral na radiografia simples ou na CT é característica de espondilodiscite por Candida $s p .^{13}$. O diagnóstico definitivo deve ser feito, de qualquer forma, por microbiologia, através de cultura da lesão observada. Deve-se começar a biópsia por agulha, e se for negativo deve ser repetido, e se essa também for negativa deve-ser feita uma biópsia aberta ${ }^{14}$. Todos os tecidos da biópsia, estudos específicos para fungos e micobactérias, junto com a cultura de rotina devem ser pedidos para esses pacientes.

O tratamento da espondilodiscite por Candida costuma ser iniciado com fluconazol. Contudo, alguns autores ${ }^{14}$ recomendam começar com anfoterecina $B$ e depois de 4-6 semanas preferem passar para fluconazol.

O prognóstico dos pacientes é muito bom, sendo que a grande maioria fica sem seqüelas e há uma taxa de cura de por volta de $82 \%$.

\section{CONCLUSÃO}

A espondilodiscite por Candida Sp é uma afecção rara. É necessário haver suspeita clínica quando há dor lombar de origem crônica com algum fator de risco associado como: uso cateteres centrais, 
uso de antibióticos de amplo espectro, uso de medicações imunossupressoras, doenças neoplásicas, abuso de substâncias endovenosas e qualquer outro tipo de imunosupressão.

Valiengo LCL, Petitto CE, Sawada JR, Fontes RBV, Pinto FCG. Spondilodiscit caused by Candida parapsilosis: Case report and literature review. Rev Med (São Paulo). 2007 abr.jun.;86(2):112-6.

\begin{abstract}
Introduction: Fungic Spondilodiscit decorrent of Candida generus species are rare, being less than 75 cases described until 2004. Case Report: Man, 42 years old, with 35 years history of alcohol abuse, was admitted with a lower abdomen pain with irradiation to inferior members and weakness of them in the past 3 months. The physical exam were: gluteus muscular atrophy, mobilization pain of the coxo-femoral articulation, L4 and L5 back pain and muscle strength grade II in the inferior member distal part. The patient was admitted and initiated clindamicin and ciprofloxacin with the suspect of spondilodiscit, confirmed by Magnetic Ressonance (RM). Two weeks latter the patient started a breathing pain. Chest radiography and computadorizated tomography shown a difuse multinodule pulmonary presentation suggestive of fungic infection or atypic tubercular presentation. Biopsy shown a granuloma with central necrosis and the culture Candida albicans. One month of antibioticotherapy there was an improvement of inferior abdomen pain and inferior members therefore without radiologic improvement. Aspirative biopsy of the lumbar mass was made with positive culture for parapsilosis candida, introducing Anfotericin B. Five months after the continuous use of these drug there were pulmonary and lumbar spine radiologic improvement with symptoms remission. Discussion: The abusive use of alcohol constitute a risk factor to the development of spondilodiscit of the Candida generus, considered that theorically improve predisposition for systemic infections by regular low virulency organisms. Conclusion: Spondilodisct caused by Cândida is a rare event, therefore must be suspected in patients with some kind of imunossupression, like severe etilism.
\end{abstract}

KEY WORDS: Discitis. Immunosuppression. Alcoholism. Case studies. Review literature. Candida/classification. Candida/pathogenicity.

\title{
REFERÊNCIAS
}

1. Kao AS, Brandt ME, Pruitt WR. The epidemiology of candidemia in two United States cities: results of population-based active surveillance. Clin Infect Dis. 1999;29:1164-70.

2. Beck-Sague CM, Jarvis WR and the National Nosocomial Infections Surveillance System. Secular trends in the epidemiology of nosocomial fungal infections in the United States, 1980-1990. J Infect Dis. 1993;167:1247-51.

3. Edwards JE. Invasive Candida infections. Evolution of a fungal pathogen. N Engl J Med. 1991;324:100-2.

4. Roca RP, Yoshikawa TT. Primary skeletal infections in heroin users: a clinical characterization, diagnosis and therapy. Clin Orthopaed Rel Res. 1979;144:238-48.

5. Ghannoum MA, Rice LB. Antifungal agents: mode of action, mechanisms of resistance, and correlation of these mechanisms with bacterial resistance. Clin Microbiol Rev. 1999;12:501-17.

6. Jonnalagadda S, Veerabagu MP, Rakela J, Kusne S, Randawa P, Rabinovitz M. Candida albicans osteomyelitis in a liver transplant recipient: a case report and review of the literature. Transplantation. 1996;62:1182-4.

7. Neale TJ, Muir JC, Mills H, Horne HJG, Jones MR.
Candida albicans vertebral osteomyelitis in chronic renal falilure. Postgrad Med J. 1987;63:695-8.

8. Lafont A, Olive A, Gelman M, Roca-Burniols J, Cots R, Carbonell J. Candida albicans spondylodiscitis and vertebral osteomyelitis in patients with intravenous heroin drug addiction: report of three cases. J Rheumatol. 1994;21:953.

9. Ferra C, Doebbeling BN, Hollis RJ, Pfaller MA, Lee CK, Gingrich RD. Candida tropicalis vertebral osteomyelitis: a late seqüela of fungemia. Clin Infect Dis. 1994;19:697703.

10. Sapico FL, Montgomerie JZ. Vertebral osteomyelitis. Infect Dis Clin North Am. 1990;4:539-50.

11. Wiley AM, Trueta J. The vascular anatomy of the spine and its relationship to pyogenic vertebral osteomyelitis. J Bone Joint Surg. 1959;41B:796-809.

12. Lew DP, Waldvogel FA. Osteomyelitis. N Engl J Med. 1997;336:999-1007.

13. Tang C. Successful treatment of Candida albicans osteomyelitis with fluconazole. J Infect. 1993;26:89-92.

14. Garbino J, Schnyder I, Lew D, Bouchuiguir-Wafa K, Rohner P. An usual cause of vertebral osteomyelitis: Candida Species. Scand J Infect Dis. 2003;35:288-91. 